\title{
Numerical investigation of compaction of deformable particles with bonded-particle model
}

\author{
Maksym Dosta ${ }^{1, *}$, Clara Costa $^{2}$, and Hazim Al-Qureshi ${ }^{2}$ \\ ${ }^{1}$ Hamburg University of Technology, 21073 Hamburg, Germany \\ ${ }^{2}$ Federal University of Santa Catarina, 89218-000 Joinville, Brazil
}

\begin{abstract}
In this contribution, a novel approach developed for the microscale modelling of particles which undergo large deformations is presented. The proposed method is based on the bonded-particle model (BPM) and multi-stage strategy to adjust material and model parameters. By the BPM, modelled objects are represented as agglomerates which consist of smaller ideally spherical particles and are connected with cylindrical solid bonds. Each bond is considered as a separate object and in each time step the forces and moments acting in them are calculated. The developed approach has been applied to simulate the compaction of elastomeric rubber particles as single particles or in a random packing. To describe the complex mechanical behaviour of the particles, the solid bonds were modelled as ideally elastic beams. The functional parameters of solid bonds as well as material parameters of bonds and primary particles were estimated based on the experimental data for rubber spheres. Obtained results for acting force and for particle deformations during uniaxial compression are in good agreement with experimental data at higher strains.
\end{abstract}

\section{Introduction}

\subsection{State of the art}

The process of powder compaction can be subdivided into the three main steps. In the first step, particles are subject to sliding and rearrangement without deformation and agglomerate breakage. The second step involves a great amount of plastic or elastic deformation and fragmentation at high pressures, filling the green body voids. Finally, in the last step the compact usually shows a near asymptotic behaviour, where the load transfers due to friction limits densification [1].

The particle behaviour during compaction is a collective result of the discrete interparticle interactions, being affected by individual particle properties, such as hardness, roughness and surface energy. In order to understand the mechanical properties of a compact and the process itself, better knowledge of relationships between individual properties and the macro behaviour is needed. Various approaches in the literature are based on continuum mechanics, as the model proposed by AlQureshi et al. [2,3], where large-deformation solution for the axisymmetric problem is proposed, even including work hardening of metallic powders. However, those models act only at the bulk scale, being phenomenological in nature. Those models cannot relate the macroscopic response of a compact to the microscopic inter-particle interaction information, such as particle rearrangement in the first step of compaction [4].

To describe a compaction process on the microscale the Discrete Element Method (DEM) can be used. This simulation method is extensively used in science and industry in a wide range of applications. In the DEM each particle is considered as a separate element and for each particle Newtonian equations of motion are solved. To calculate interparticle interactions hard and soft sphere types of models can be employed. In the case of the soft sphere model it is supposed that particles can undergo small virtual deformations. Based on these values and interparticle overlaps the contact forces are calculated. However, all stressed particles are still treated as ideally spherical objects without considering of shape modification. Moreover, most of contact models are based on the theory of Hertz, which can be applied for relative small deformations. Therefore, the DEM cannot be directly applied for modelling of second of third steps of compaction.

Many different methods have been proposed in the literature, such as Olson and Larsson [5], which use micromechanical tests and FEM to calibrate the particle properties and contact formulations. These were then implemented in the DEM. Hathong et al. [6] have used the Meshed Discrete Element Method (MDEM), in which elements are deformable for small-scale experiments, yielding effective properties used on a fullscale simulation with the DEM. Jerier et al. [7] modified the DEM at high densifications $(>85 \%)$ during a powder

\footnotetext{
Corresponding author: dosta@tuhh.de
} 
compaction by using contact models derived from FEM simulations, using Voronoi cells. A similar approach was also used by Zhang [8], Frenning [9] and Güner et al. [10].

However, despite various research works performed in this area, there is no efficient way to perform simulation of compaction on the microscale. In this contribution the novel modelling approach based on the Bonded-ParticleModel (BPM) is presented and its advantages and disadvantages are discussed.

\subsection{Bonded-Particle Model (BPM)}

The BPM is an extension of the DEM, where each two primary particles can be connected by solid or liquid bonds [11-14]. This allows to create spherical or irregular-shaped objects as aggregates consisting of primary particles connected with bonds [13]. Moreover, it allows to simulate elasto-plastic deformation or even breakage of modelled objects.

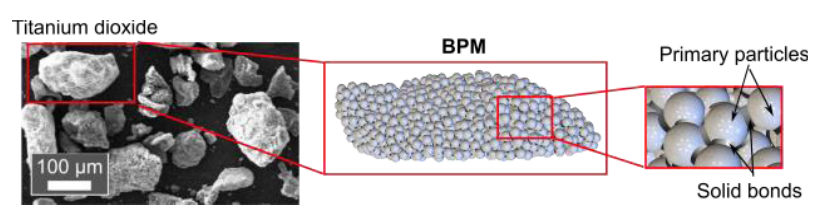

Fig. 1. Representation of titanium dioxide with BPM.

In this contribution the BPM was used to simulate behaviour of spherical rubber particles. Solid bonds between primary particles were described with elastic solid beam model [12]. In each time step, based on the deformations of bonds, the forces and moments acting on primary particles were estimated. As a result, the relative movement of primary particles and deformation of initial objects were calculated.

To perform a simulation of compaction, the BPM was implemented into component-based simulation framework MUSEN [14].

\section{Parameter Estimation}

The spherical rubber particles of diameter $2 \mathrm{~cm}$ have been used as model material in this contribution. The rubber materials are thermoset polymers known for their elastic behaviour and ability to withstand extensive deformation and to return to the original state once the applied force is removed.

Experimental data previously obtained by Shima et al. [15] have been used as reference values. In their experiments the spherical particles were uniaxial compressed and resulted compressive force and lateral deformation were measured, as it is schematically shown in Fig. 2. The force was measured by the load cell and the total displacement by a displacement meter. The starting compression point is visually detected when the rubber and the plate start touching each other. In the reference experiment the lateral extension $(U(R))$ for the diameter of the spheres was measured using photography at each displacement level, measuring at each force level the expended displacement of the horizontal diameter and taking half of the total as the lateral extension. During the experiment the radius of contact surface (a) between the rubber sphere and the plate was measured through a white paper on the bottom of the sample that was coloured during the compression due to a red ink on the rubber.

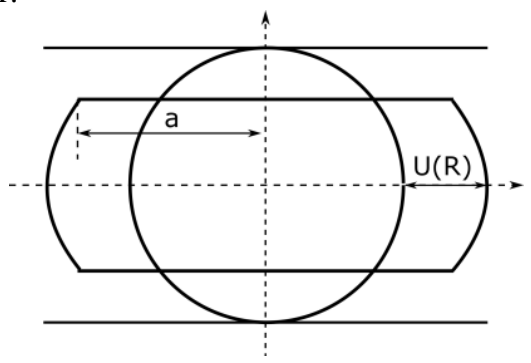

Fig. 2. Schematic representation of performed experiment.

In order to simulate complex behaviour of the rubber sphere a unimodal distribution of primary particles connected by solid bonds was chosen. To evaluate applicability of the proposed modelling approach, the two different shape discretizations were analysed (see Tab. 1). On the one hand a large number of primary particles allows to get better shape representation, on the other hand it leads to much higher computational effort.

Table 1. Representation of rubber sphere with BPM.

\begin{tabular}{|l|c|c|}
\hline & Fine & Coarse \\
\hline Primary particles & 1171 & 93 \\
\hline $\begin{array}{l}\text { Diameter of primary } \\
\text { particle [mm] }\end{array}$ & 1.6 & 3.5 \\
\hline Solid bonds & 8135 & 303 \\
\hline Bond diameter [mm] & 1.28 & 2.8 \\
\hline
\end{tabular}

Material parameters like density, Poisson ratio or friction coefficients of primary particles and bonds was taken equal to material parameters of rubber. However, due to the modifications of a structural model (transition from solid rubber particle to agglomerate) Young's moduli of primary particles $E_{p}$ and bonds $E_{b}$ were used as adjustment parameters. For fine approximation were used: $\mathrm{E}_{\mathrm{p}}=10.8 \mathrm{MPa}$ and $\mathrm{E}_{\mathrm{b}}=0.2 \mathrm{MPa}$, for coarse: $\mathrm{E}_{\mathrm{p}}=17.6$ $\mathrm{MPa}$ and $\mathrm{E}_{\mathrm{b}}=0.37 \mathrm{MPa}$.

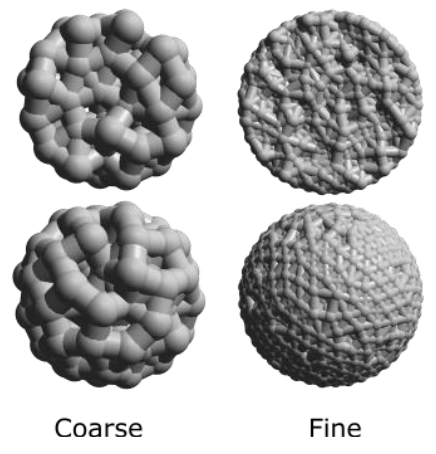

Fig. 3. Coarse and fine representation of rubber sphere.

Some materials such as elastomers have a different behaviour in tension or compression [16]. In order to reproduce such behaviour a solid bond model was extended with an additional parameter to describe 
bimodularity. This parameter defines the difference between effective Young's moduli of bond in tension and compression [16].

\section{Simulation results}

\subsection{Single particle compression}

In order to adjust model parameters and to validate the proposed approach, uniaxial compression of a single agglomerate was employed. The investigated aggregate was placed between the two walls, whereas the upper wall was moved with predefined velocity, as it is schematically shown in Fig. 4. To avoid oscillations caused in result of wave propagation in material, the slow compression velocity was chosen.

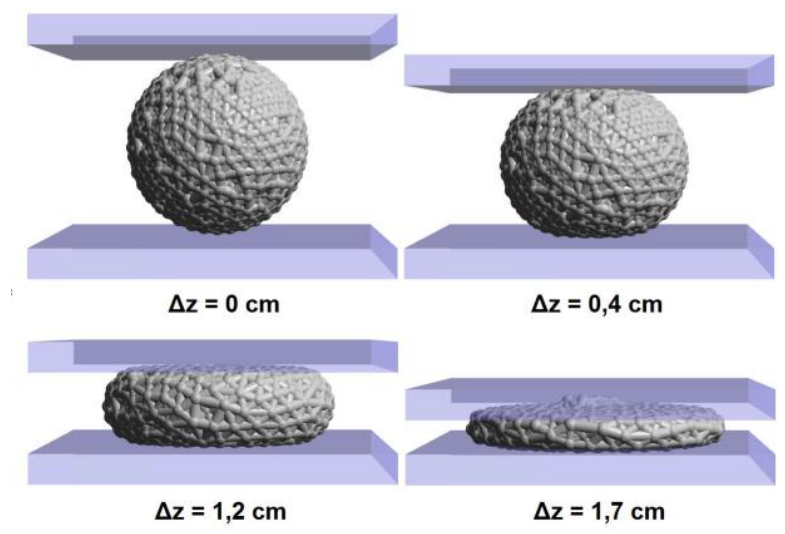

Fig. 4. Compression of single rubber particle.

In Fig. 5 and Fig. 6 main characteristics obtained from simulation studies for fine and coarse shape approximations are shown. In both figures the increase of the compression force $F$ and radial elongation $U(R)$ are represented. To see the influence of initial placement of primary particles several aggregates of each type were modelled. The deviations of simulation results are shown with error bars in Fig. 5 and Fig. 6. Moreover, it can be seen there exist differences between results obtained from coarse and fine representation, as well as coarse representation leads to larger deviations. This is caused due to higher material anisotropy caused due to representation of spherical shape only with 93 primary particles.

From the obtained results the conclusion can be drawn, that in compare to the Hertz model the BPM representation allows to predict correctly the force as well as the deformation of objects. Moreover, even a coarse description of an agglomerate shape allows to describe the deformation correctly. The Hertz model is not applicable for large deformations, therefore for strain greater than $10 \%$ it is visualized as dotted line.
It is to note, however, that the proposed model allows to describe only those mechanical deformation of spherical objects for strains that are smaller than $50 \%$. For larger strains, the BPM model is not able to describe objects behaviour.

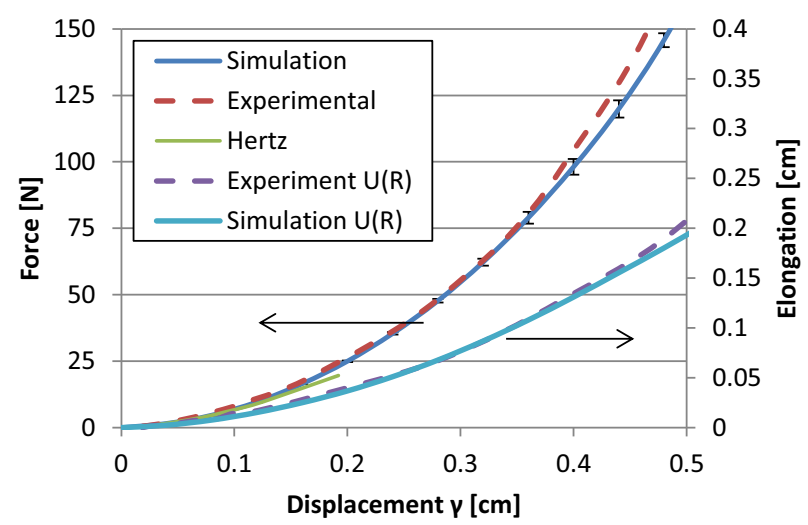

Fig. 5. Fine representation (1171 primary particles).

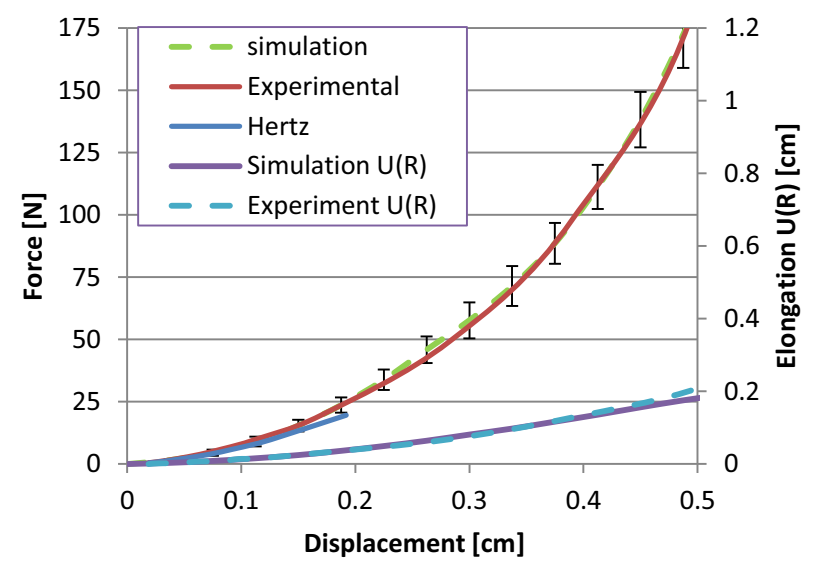

Fig. 6. Coarse representation (93 primary particles).

\subsection{Compression of particle collective}

After fitting of the parameters for the BPM, the packing consisting of about 2500 ideally spherical rubber particles was modelled without considering of particle deformation. For this purpose Hertz-Mindlin model was used. The particles were filled into cylindrical volume. This packing was uniaxially compressed and the obtained behaviour was compared to the behaviour of the similar packing consisting of coarse aggregates (deformable particles) as it is schematically shown in Fig 7.

In Fig. 8 the obtained force-displacement characteristics for both cases is shown. Here, the forces acting on upper and bottom plates are presented. It can be seen, that there is a decisive difference between simulation results, where the non-deformable spherical particles were modelled by using the Hertz-Mindlin model [12] and 
where deformable agglomerates were modelled by the BPM. Due to a set of simplifications in the case of spherical particles, like application of the Hertz theory and negligence of deformation, there is an underprediction of the force for the large strains.

However, it should be mentioned, that a coarse shape representation in the BPM, results in overpredicted initial packing density. This is caused due to penetration of primary particles of agglomerate into voids between primary particles of neighbour agglomerates. Therefore, for the detailed investigation of a compaction process a better shape representation and a higher amount of primary particles are needed.

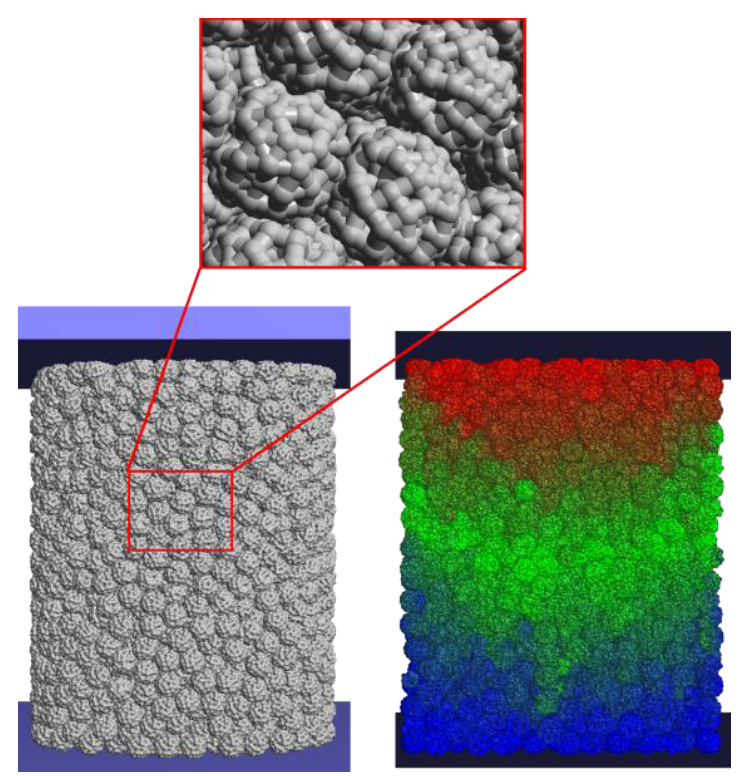

Fig. 7. Compression of particle collective consisting of coarse aggregates (Right side: particles are coloured according to their velocity).

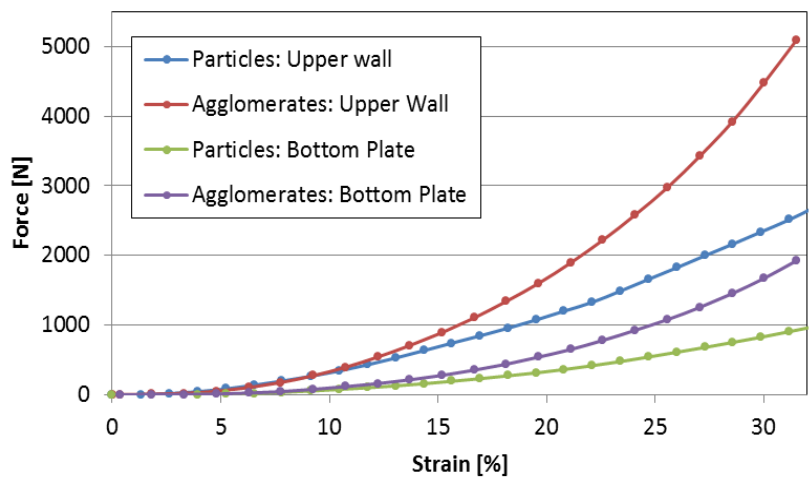

Fig. 8. Force-displacement characteristics obtained from compression of rubber particles.

\section{Conclusions}

In this contribution the application of the Bonded Particle Model for numerical investigation of compaction process was proposed. This technique was validated on experimental results obtained from uniaxial compression of a single rubber particle. From the results it is apparent that in compare to the convenient DEM, this technique is a very effective way to describe large deformations of objects caused in result of compressive stresses. However, for a precise description of particle shapes fine discretization should be employed.

Application of proposed technique for particle collective showed that considering the particle deformation leads, as expected, to higher compressive forces. However, for the further model additional validation experiments are needed and, therefore, should be performed in future.

\section{References}

1. A. Samimi, A. Hassanpour, M. Ghadiri. CES 60, 3993-4004, (2005).

2. H.A. Al-Qureshi, M.R.F. Soares, D. Hotza, M.C. Alves, A.N. Klein. J. of Materials Processing Techn. 199, 417-424, (2008).

3. H.A. Al-Qureshi, A. Galiotto, A.N. Klein. J. of Materials Processing Techn. 166, 135-143, (2005).

4. H.P. Zhu, Z.Y. Zhou, R.Y. Yang, A.B. Yu. CES 63, 5728-5770, (2008).

5. E. Olsson, P.-L. Larsson. Powd. Techn. 243, 71-78 (2013).

6. B. Hathong, J.-F. Jerier, P. Doremus, D. Imbault, F.V. Donze. Int. J. of Solids and Structures 46, 33573364, (2009).

7. J.-F. Jerier, B. Hathong, V. Richefeu, B. Chareyre, D. Imbault, F.-V. Donze, P. Doremus. Powd. Techn., 208, 537-541, (2011).

8. J. Zhang. Composite Sci. and Techn., 62, 20482053, (2009).

9. G. Frenning. CES 65, 2464-2471, (2010).

10. F. Güner, Ö.N. Cora, H. Sofuoglu. Powd. Techn., 271, 238-247, (2015).

11. D.O. Potyondy. Geosystem Eng. 18, 1-28 (2015).

12. M. Dosta, S. Dale, C.R. Wassgren, S. Heinrich, J.D. Litster. Powd. Techn. 299, 87-97 (2016).

13. S. Kozhar, M. Dosta, S. Antonyuk, S. Heinrich, L. Gilson, U. Bröckel. Adv. Powd. Techn. 26, 767-777 (2015).

14. M. Dosta, S. Antonyuk, S. Heinrich. IECr 52, 11275-11281 (2013).

15. S. Shima, Y. Tatara, M. Lio, J.C. Lucero. JSME Int. J. 36, 197-205 (1993).

16. M. Destrade, J.G. Murphy, B. Rashid. Soft Cond. Matter. 1, 29-37 (2009). 